\title{
ON THE FRATTINI SUBGROUP OF THE GENERALIZED FREE PRODUCT WITH AMALGAMATION
}

\author{
D. Ž. DJOKOVIĆ AND C. Y. TANG ${ }^{1}$
}

\begin{abstract}
AbSTRACr. Let $G$ be the generalized free product of two groups $A$ and $B$ amalgamating their subgroup $H$. If $H$ satisfies the minimal condition on subgroups then the Frattini subgroup of $G$ is contained in $H$. When $H$ is finite then this Frattini subgroup can be precisely determined.
\end{abstract}

1. Notation. Our notation is standard: $H \leqq G$ means $H$ is a subgroup of $G ; H<G$ is used for proper subgroups; $H \triangleleft G$ for normal subgroups.

By $G=(A * B)_{H}$ we denote the generalized free product of $A$ and $B$ amalgamating their common subgroup $H$.

If $x \in G \backslash H$ then $x$ has exactly one of the following forms:

(1) $x=a_{1} b_{1} a_{2} b_{2} \cdots a_{m}$,

(2) $x=a_{1} b_{1} a_{2} b_{2} \cdots a_{m} b_{m}$,

(3) $x=b_{1} a_{1} b_{2} a_{2} \cdots b_{m} a_{m}$,

(4) $x=b_{1} a_{1} b_{2} a_{2} \cdots b_{m}$,

where $m$ is a positive integer and $a_{i} \in A \backslash H, b_{i} \in B \backslash H$.

We shall write $x \in{ }_{A} W$ if $x$ has the form (1) or (2). Similarly $x \in{ }_{B} W$ if $x$ has the form (3) or (4). Also, $x \in W_{A}$ if $x$ has form (1) or (3) and $x \in W_{B}$ if $x$ has form (2) or (4).

By $\Phi(G)$ we denote the Frattini subgroup of a group $G$.

2. Main results. By an easy application of Zorn's lemma one can show that if $H \leqq G$ there exists a unique maximal normal subgroup of $G$ contained in $H$. More precisely this subgroup, say $K(H, G)$, is characterized by the following properties:

(i) $K(H, G) \triangleleft G$,

(ii) $K(H, G) \leqq H$,

(iii) $X \triangleleft G \& X \leqq H \Rightarrow X \leqq K(H, G)$.

It is immediate that for $x \in G$ the following holds:

(5) $x \in K(H, G) \Leftrightarrow(\forall y \in G) y^{-1} x y \in H$.

Received by the editors November 12, 1970.

AMS 1970 subject classifications. Primary 20E20, 20E30; Secondary $20 \mathrm{~F} 30$.

Key words and phrases. Group, Frattini subgroup, generalized free product with amalgamation, minimal condition.

${ }^{1}$ The work of the first author was supported in part by NRC Grant A-5285, and the work of the second author by NRC Grant A-4064.

(c) American Mathematical Society 1972 
The following lemma is due to A. Whittemore [2, Proposition 2.3].

LEMMA 1. If $G=(A * B)_{H}$ and there exists $x \in G$ such that $H \cap x H x^{-1}=1$ then $\Phi(G)=1$.

Our main result is

THEOREM 1. If $G=(A * B)_{H}, K(H, G)=1$, and $H$ satisfies the minimum condition on subgroups then $\Phi(G)=1$.

Proof. Graham Higman and B. H. Neumann have shown [1] that $H=1$ implies $\Phi(G)=1$. Thus we shall assume that $H \neq 1$.

Consider the set of all subgroups of $G$ of the form $H \cap x H x^{-1}, x \in G$. By our minimal condition there exists a minimal element in this set, say $M=H \cap u H u^{-1}$. Since $K(H, G)=1$ and $H \neq 1$ it follows that $M$ is a proper subgroup of $H$ and consequently $u \notin H$, say $u \in W_{A}$.

Assume that $M \neq 1$ and choose $y \in M, y \neq 1$. Then $u^{-1} y u \in H$. It will be shown in Lemma 2 that there exists $v \in_{B} W$ such that $v^{-1} u^{-1} y u v \notin H$.

If $h \in H$ then

$$
h \notin M \Leftrightarrow u^{-1} h u \notin H \Rightarrow v^{-1} u^{-1} h u v \notin H \Leftrightarrow h \notin(u v) H(u v)^{-1} .
$$

Hence $H \cap(u v) H(u v)^{-1}$ is a subgroup of $M$. It is a proper subgroup of $M$ because $y \in M$ and $y \notin(u v) H(u v)^{-1}$. This contradicts our choice of $M$. Therefore we must have $M=1$ and the assertion follows from Lemma 1 .

It remains to prove the next lemma which asserts a little bit more than we used in the proof of Theorem 1.

Lemma 2. Let $G=(A * B)_{H}, K(H, G)=1$ and assume that $H$ satisfies the minimum condition on subgroups normal in $B$. Then for every $h \in H$, $h \neq 1$, there exists $w \in_{B} W$ such that $w^{-1} h w \notin H$.

Proof. Let $H=H_{0}$ and define

$$
\begin{array}{rlrl}
H_{2 i+1} & =K\left(H_{2 i}, A\right), & i \geqq 0, \\
H_{2 k} & =K\left(H_{2 k-1}, B\right), \quad k \geqq 1 .
\end{array}
$$

We have $H_{0} \geqq H_{1} \geqq H_{2} \geqq \cdots$, and $\bigcap_{i \geqq 0} H_{i}=1$ because $K(H, G)=1$. Our minimum condition implies that $H_{n}=1$ for large $n$. Let $m$ be the smallest integer such that $H_{m}=1$. Then we have

$$
H_{0} \geqq H_{1}>H_{2}>\cdots>H_{m}=1 \text {. }
$$

Let $j$ be the unique integer such that $h \in H_{j} \backslash H_{j+1}$. We shall distinguish two cases:

Case 1. $j=2 k-1$ is odd. Then by (5) there exists $b_{1} \in B \backslash H$ such that $b_{1}^{-1} h b_{1} \in H_{2 k-2} \backslash H_{2 k-1}$. Now, there exists $a_{1} \in A \backslash H$ such that $a_{1}^{-1} b_{1}^{-1} h b_{1} a_{1} \in$ 
$H_{2 k-3} \mid H_{2 k-2}$. Continuing in this manner we get $w=b_{1} a_{1} \cdots \in_{B} W$ with the property that $w^{-1} h w \notin H$.

Case 2. $j=2 i$ is even. Take $b_{1} \in B \backslash H$ and consider $b_{1}^{-1} h b_{1}$. If $b_{1}^{-1} h b_{1} \in$ $\left.H_{2 i}\right) H_{2 i+1}$ then we can continue as in Case 1.

Suppose that $b_{1}^{-1} h b_{1} \in H_{2 i+1} \backslash H_{2 i+2}$. Then we take $a_{1} \in A \backslash H$ and consider $a_{1}^{-1} b_{1}^{-1} h b_{1} a_{1}$. If $a_{1}^{-1} b_{1}^{-1} h b_{1} a_{1} \in H_{2 i+1} \backslash H_{2 i+2}$ we can continue as in Case 1 . If $a_{1}^{-1} b_{1}^{-1} h b_{1} a_{1} \in H_{2 i+2} \backslash H_{2 i+3}$ then we transform this element by $b_{2} \in B \backslash H$. It is clear that because the sequence (6) is finite the Case 2 after a finite number of such steps must reduce to Case 1 .

The lemma is proved.

It follows from Theorem 1 that if $G=(A * B)_{H}$, where $H$ is finite, then $\Phi(G) \leqq K(H, G)$.

\section{REFERENCES}

1. G. Higman and B. H. Neumann, On two questions of Itô, J. London Math. Soc. 29 (1954), 84-88. MR 15, 286.

2. A. Whittemore, On the Frattini subgroup, Trans. Amer. Math. Soc. 141 (1969), 323-333. MR 39 \#6993.

Department of Pure Mathematics, University of Waterloo, Waterloo, Ontario, Canada 